\section{Taking cancer imaging up a PEG}

\section{By Joanne Kotz, Senior Editor}

Researchers at The Johns Hopkins University and Virginia Commonwealth University have developed a new method for imaging primary and metastatic tumors that may be broadly useful across multiple cancers. ${ }^{1}$ The lead investigators have founded a company, Cancer Targeting Systems Inc., that plans to move the imaging approach into patients.

The mainstays of clinical cancer imaging are MRI, X-ray CT and PET.

MRI and CT scans are easy to use and do not require injecting anything into the patient prior to imaging. However, these scans only provide information on tissue structure and thus cannot always unambiguously identify tumors. For example, it can be difficult to distinguish between tumor tissue and scar tissue on a CT scan.

$\left[{ }^{18} \mathrm{~F}\right]$ fluorodeoxyglucose (FDG) PET provides functional information that can be useful for staging cancers or clarifying MRI or CT results. The procedure involves injecting patients with the radioactive sugar analog FDG, which is preferentially taken up by cancer cells because of their higher metabolic rate. The distribution of radioactivity in the patient is then measured with a PET scan.

FDG PET is "sensitive but not specific for cancer" because there can be background from other tissues with high metabolic rates including heart, brain, muscle and fatty tissue, said Martin Pomper, professor of radiology at Johns Hopkins. Diseases other than cancer, such as inflammation or infection, can also take up FDG and appear bright on PET scans, he added.

Thus, he said, "the push now is to develop imaging approaches that are more unique to cancer."

More targeted approaches in preclinical or clinical development image cancer-related processes such as angiogenesis or cancer-specific biomarkers. However, these approaches only work in a subset of cancers or tumor sites. As a result, none is likely to be useful across all cancers.

Pomper, therefore, set out to find "a method that was both specific to cancer but nonspecific as to the type or site of the cancer."

He teamed up with Paul Fisher, professor of human and molecular genetics at VCU and director of the VCU Institute of Molecular Medicine. In 1997, Fisher discovered a cancer-specific promoter upstream of the gene encoding the rodent protein Peg-3 (protein phosphatase 1 regulatory inhibitor subunit $15 \mathrm{~A}$; Ppp1r15a). ${ }^{2}$
In a paper published in 2005, Fisher showed that the Peg-3 promoter, when transfected into human cells, was activated in cancer cells but not in noncancerous cells. ${ }^{3}$

Now, Pomper and Fisher have shown that this cancer-specific promoter can be used to image human tumors.

The researchers first constructed a plasmid in which the Peg-3 promoter regulated expression of HSV1-TK, a reporter gene that is used in conjunction with a radionuclide tracer in PET or a related radioactivity-based imaging approach called single-photon emission computed tomography (SPECT).

Next, the team used a nonviral delivery vehicle and injected the plasmid into mice with human metastatic melanoma, followed 46 hours later by injection of the radiotracer. Subsequent SPECT imaging showed that the radioactive signal at a metastatic site in the lungs of mice with melanoma was 31-fold higher than the background signal in the lungs of healthy animals.

Metastatic lesions also were detected in the dorsal neck, spinal cord, diaphragm and lymph nodes.

Finally, the researchers compared their Peg-3 promoter-based imaging approach with FDG PET.

In mouse models of human melanoma and human breast cancer, both methods detected the primary tumor and most metastatic sites. However, metastatic sites adjacent to the heart and brown fat tissue, which are difficult to detect with FDG PET because these metabolically active tissues give high background signals, were better detected by Peg-3-based imaging.

Data were published in Nature Medicine.

"These preclinical data are really encouraging," said John Joyal, VP of discovery research at Molecular Insight Pharmaceuticals Inc. He said the findings suggest that Peg-3-based imaging has potential applications in diagnostics, prognostics, staging and guiding cancer treatment.

"This is an extremely interesting development," added Peter Choyke, chief of the molecular imaging program at the National Cancer Institute. The findings "could provide a completely unique imaging biomarker of specific transcription factors found in some human tumors," he said.

According to Choyke, the main limitation with Peg-3 imaging "is its potential complexity compared with current methods, which typically involve only one injection of a contrast agent and do not require gene transfection. Here, the patient would undergo a prepping transfection of the Peg-3 promoter with HSV1-TK, followed by injection of a radionuclide several days later."

Thus, he said, "the real question about potential clinical applications is whether a signal from a tumor has clinical meaning above seeing the tumor on a CT scan or detecting glucose metabolism on a standard FDG PET scan."

If it is just another way to see a tumor, Choyke said, even though it may offer potential advantages such as seeing metastases near 


\section{ANALYSIS}

metabolically active sites, it will have trouble competing with significantly less complex imaging methods.

Choyke said that an imaging method based on gene transfection will likely need to be combined with treatment in some fashion to offer enough of an advantage to have clinical impact. Thus an important next direction will be "the development of a plausible gene therapy approach based on the Peg-3 promoter" for use as a theranostic, he said.

In contrast, Joyal sees a significant opportunity for using the Peg-3 promoter in combination with a reporter gene strictly as an imaging agent and, if appropriately modified, potentially as a theranostic as well.

FDG PET and other targeted imaging agents each work better for particular tumor types, Joyal told SciBX. Therefore, Peg-3-based imaging has the potential to be a "more universal cancer imaging agent," he said.

Molecular Insight's lead molecular imaging agent is Trofex (MIP1072), a ${ }^{123}$ I-labeled small molecule inhibitor of prostate-specific membrane antigen (PSMA). Trofex is in Phase I testing to diagnose and monitor metastatic prostate cancer.

Joyal said the most critical next step will be determining whether cancer-specific activation of the Peg-3 promoter occurs in humans similarly to how it occurs in mice with human cancer cell lines.

\section{Peg race}

Cancer Targeting Systems is hoping to move Peg-3-based imaging to the clinic as fast as possible.

"We intend to test a few other tumor models and try some other nonviral delivery vehicles," said Pomper. "Then we will pick the best indication and the best vehicle and begin discussions with the FDA to learn what sort of toxicity package will be needed to administer this to human subjects. The key hurdle will come in the gene itself, and we will need to engage the FDA to discuss carefully what we need to do to translate these results."

Detecting metastases to stage cancer is the first application that Cancer Targeting Systems will focus on. The biotech also hopes to develop theranostic tools that combine Peg-3 imaging and gene therapy, said Pomper.

Cancer-specific expression of the Peg-3 promoter and its use in gene therapy are covered by patents held by Columbia University, where Fisher was located when he discovered the promoter. Johns Hopkins has filed for a patent covering the Peg-3-based imaging and theranostic applications reported in the Nature Medicine paper.

Pomper and Fisher are in ongoing discussions with Johns Hopkins and Columbia to license the relevant patents to Cancer Targeting Systems and are seeking funding and partners, said Pomper.

Kotz, J. SciBX 4(2); doi:10.1038/scibx.2011.32

Published online Jan. 13, 2011

\section{REFERENCES}

1. Bhang, H.-e.C. et al. Nat. Med.; published online Dec. 12, 2010; doi:10.1038/nm.2269

Contact: Martin G. Pomper, Johns Hopkins Medical Institutions, Baltimore, Md.

e-mail: mpomper@jhmi.edu

2. Su, Z.-Z. et al. Proc. Natl. Acad. Sci. USA 94, 9125-9130 (1997)

3. Su, Z.-Z. et al. Proc. Natl. Acad. Sci. USA 102, 1059-1064 (2005)

\section{COMPANIES AND INSTITUTIONS MENTIONED}

Cancer Targeting Systems Inc., no location yet

Columbia University, New York, N.Y.

The Johns Hopkins University, Baltimore, Md.

Molecular Insight Pharmaceuticals Inc. (OTCQB:MIPIQ),

Cambridge, Mass.

National Cancer Institute, Bethesda, Md.

Virginia Commonwealth University, Richmond, Va. 